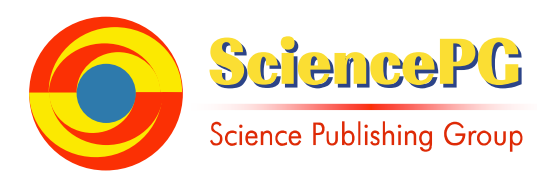

\title{
Krylov-Bogoliubov-Mitropolskii Method for Fourth Order More Critically Damped Nonlinear Systems
}

\author{
Md. Mahafujur Rahaman \\ Department of Computer Science \& Engineering, Z.H. Sikder University of Science \& Technology, Shariatpur, Bangladesh
}

\section{Email address:}

mahfuz0809@gmail.com

\section{To cite this article:}

Md. Mahafujur Rahaman. Krylov-Bogoliubov-Mitropolskii Method for Fourth Order More Critically Damped Nonlinear Systems. American Journal of Applied Mathematics. Vol. 3, No. 6, 2015, pp. 265-270. doi: 10.11648/j.ajam.20150306.15

\begin{abstract}
With a view to obtaining the transient response of the system where triply eigenvalues are equal and another is distinct, we have considered a fourth order more critically damped nonlinear systems, and enquired into analytical approximate solution in this paper. We have also suggested that the results obtained by the proposed method correspond to the numerical solutions obtained by the fourth order Runge-Kutta method satisfactorily.
\end{abstract}

Keywords: KBM, Eigenvalues, More Critically Damped System, Nonlinearity, Runge-Kutta Method

\section{Introduction}

The KBM [1, 2] method is a broadly exercised technique to study nonlinear oscillatory and non-oscillatory differential systems with small nonlinearities. Initially, the method was developed by Krylov and Bogoliubov [2] for finding the periodic solutions of second order nonlinear differential systems with small nonlinearities. Later on, the method was improved and justified mathematically by Bogoliubov and Mitroposkii [1]. Popov [3] extended the method to damped oscillatory nonlinear systems. Due to physical importance of the damped oscillatory systems, Popov's results were rediscovered by Mendelson [4]. Then, this method was extended by Murty and Deekshatulu [5] for over-damped nonlinear systems. Sattar [6] studied the second order critically-damped nonlinear systems by using of the KBM method. Murty [7] proposed a unified KBM method for second order nonlinear systems which covers the undamped, over-damped and damped oscillatory cases. Next, Osiniskii [8] first developed the KBM method to solve third-order nonlinear differential systems imposing some restrictions, which made the solution over-simplified. Mulholland [9] removed these restrictions and found desired solutions of third order nonlinear systems. Bojadziv [10] assessed solutions of nonlinear systems by converting it to a three-dimensional differential system. Sattar [11] examined solutions of three-dimensional over-damped nonlinear systems. Shamsul [12] propounded an asymptotic method for second order over-damped and critically damped nonlinear systems. Shamsul [13] then extended the method presented in [12] to third order over-damped nonlinear systems under some special conditions. Akbar et al. [14] generalized the method and showed that their method was easier than the method of Murty et al. [15]. Later, Akbar et al. [16] extended the method presented in Akbar et al. [14] for fourth order damped oscillatory systems. Again, Akbar et al. [17] investigated a technique for obtaining over-damped solutions of $n$-th order nonlinear differential equations under some special conditions including the case of internal resonance. A method has been established by Akbar et al. [18] for solving the fourth order more critically damped systems. Soon after Rokibul et al. [19] expounded an analytical approximate solution of fourth order more critically damped systems when the unequal eigenvalue is integral multiple of equal eigenvalues. Afterwards Hakim [20] presented a method to enquire solutions of fourth order more critically damped nonlinear systems.

In this article, we have investigated solutions of fourth order more critically damped nonlinear systems i.e. the three eigenvalues are equal and the other one is distinct, by developing a method which is different from the method of Akbar et al. [18], Rokibul et al. [19] and Hakim [20]. Finally, in this paper, we have suggested that the acquired perturbation results show good coincidence with the numerical results for different sets of initial conditions as well as different sets of eigenvalues. 


\section{The Method}

Let us consider a weakly nonlinear fourth order ordinary differential system

$$
x^{(i v)}+k_{1} \dddot{x}+k_{2} \ddot{x}+k_{3} \dot{x}+k_{4} x=-\varepsilon f(x, \dot{x}, \ddot{x}, \dddot{x})
$$

In which $x^{(i v)}$ indicates the fourth derivative of $x$, over dots indicate the first, second and third derivatives with respect to $t ; k_{1}, k_{2}, k_{3}, k_{4}$ are characteristic parameters, $\varepsilon$ is a small parameter and $f(x, \dot{x}, \ddot{x}, \dddot{x})$ is the nonlinear function.

When $\varepsilon=0$, the equation becomes linear and the solution of the linear equation of (1) is

$$
x(t, 0)=\left(a_{0}+b_{0} t+c_{0} t^{2}\right) e^{-\lambda t}+d_{0} e^{-\mu t}
$$

In which $a_{0}, b_{0}, c_{0}$ and $d_{0}$ are constants of integration.

However, when $\varepsilon \neq 0$, following Shamsul [21], the solution of the equation (1) is sought in the form

$$
\begin{aligned}
x(t, \varepsilon)= & \left(a+b t+c t^{2}\right) e^{-\lambda t}+d e^{-\mu t} \\
& +\varepsilon u_{1}(a, b, c, d, t)+\cdots
\end{aligned}
$$

where $a, b, c$ and $d$ are slowly varying functions of time $t$ and satisfy the following first order differential equations:

$$
\begin{aligned}
& \dot{a}(t)=\varepsilon A_{1}(a, b, c, d, t)+\cdots \\
& \dot{b}(t)=\varepsilon B_{1}(a, b, c, d, t)+\cdots \\
& \dot{c}(t)=\varepsilon C_{1}(a, b, c, d, t)+\cdots \\
& \dot{d}(t)=\varepsilon D_{1}(a, b, c, d, t)+\cdots
\end{aligned}
$$

In this calculation, we have merely considered first few terms in the series expansion of (3) and (4) and we have calculated the functions $u_{i}$ and $A_{i}, B_{i}, C_{i}, D_{i}$ for $i=1,2, \cdots \cdots, n$ such that $a, b, c$ and $d$ appearing in (3) and (4) satisfy the given differential equation (1).

With a view to ascertaining these unknown functions, the KBM method usually suggests that the correction terms, $u_{i}$ for $i=1,2, \cdots \cdots, n$ should exclude terms (sometimes referred to as secular terms) that enlarge them. The solution may be, in theory, accurate for any order of approximation. But due to the rapid rise in algebraic intricacy for the derivation of the formulae, the solution is generally limited to a lower order, especially the first order (Murty [7]).

Now differentiating the equation (3) four times with respect to $t$, substituting the value of $x$ and the derivatives $\dot{x}, \ddot{x}, \dddot{x}, x^{(i v)}$ in the equation (1), using the relations presented in (4) and finally equating the coefficients of $\mathcal{E}$, we obtain

$$
\begin{gathered}
e^{-\lambda t}\left(\frac{\partial}{\partial t}+\mu-\lambda\right)\left\{\frac{\partial^{2} A_{1}}{\partial t^{2}}+3 \frac{\partial B_{1}}{\partial t}+6 C_{1}+\right. \\
\left.t\left(\frac{\partial^{2} C_{1}}{\partial t^{2}}+6 \frac{\partial C_{1}}{\partial t}\right)+t^{2} \frac{\partial^{2} C_{1}}{\partial t^{2}}\right\}+e^{-\mu t} \\
\left(\frac{\partial}{\partial t}+\lambda-\mu\right)^{3} D_{1}+\left(\frac{\partial}{\partial t}+\lambda\right)^{3}\left(\frac{\partial}{\partial t}+\mu\right) u_{1} \\
=-f^{(0)}(a, b, c, d, t)
\end{gathered}
$$

where $f^{(0)}(a, b, c, d, t)=f\left(x_{0}, \dot{x}_{0}, \ddot{x}_{0}, \dddot{x}_{0}\right)$ and $x_{0}=\left(a+b t+c t^{2}\right) e^{-\lambda t}+d e^{-\mu t}$

Now we expand $f^{(0)}$ in the Taylor's series of the form

$$
\begin{aligned}
f^{(0)} & =\left(a+b t+c t^{2}\right)^{0} \sum_{i, j=0}^{\infty} F_{0}(d) e^{-(i \lambda+j \mu) t} \\
& +\left(a+b t+c t^{2}\right)^{1} \sum_{i, j=0}^{\infty} F_{1}(d) e^{-(i \lambda+j \mu) t} \\
& +\left(a+b t+c t^{2}\right)^{2} \sum_{i, j=0}^{\infty} F_{2}(d) e^{-(i \lambda+j \mu) t} \\
& +\left(a+b t+c t^{2}\right)^{3} \sum_{i, j=0}^{\infty} F_{3}(d) e^{-(i \lambda+j \mu) t}+\cdots
\end{aligned}
$$

Thus we can write

$$
\begin{aligned}
& e^{-\lambda t}\left(\frac{\partial}{\partial t}+\mu-\lambda\right)\left\{\frac{\partial^{2} A_{1}}{\partial t^{2}}+3 \frac{\partial B_{1}}{\partial t}+6 C_{1}\right. \\
& \left.+t\left(\frac{\partial^{2} B_{1}}{\partial t^{2}}+6 \frac{\partial C_{1}}{\partial t}\right)+t^{2} \frac{\partial^{2} C_{1}}{\partial t^{2}}\right\} \\
& +e^{-\mu t}\left(\frac{\partial}{\partial t}+\lambda-\mu\right)^{3} D_{1}+\left(\frac{\partial}{\partial t}+\lambda\right)^{3} \\
& \left(\frac{\partial}{\partial t}+\mu\right) u_{1}=-\left\{\left(a+b t+c t^{2}\right)^{0}\right. \\
& \sum_{i, j=0}^{\infty} F_{0}(d) e^{-(i \lambda+j \mu) t}+\left(a+b t+c t^{2}\right)^{1} \\
& \sum_{i, j=0}^{\infty} F_{1}(d) e^{-(i \lambda+j \mu) t}+\left(a+b t+c t^{2}\right)^{2} \\
& \sum_{i, j=0}^{\infty} F_{2}(d) e^{-(i \lambda+j \mu) t}+\left(a+b t+c t^{2}\right)^{3} \\
& \left.\sum_{i, j=0}^{\infty} F_{3}(d) e^{-(i \lambda+j \mu) t}+\cdots\right\}
\end{aligned}
$$

We impose the condition that $u_{1}$ cannot contain the fundamental terms of $f^{(0)}$, therefore equation (7) can be 
separated for unknowns functions $u_{1}$ and $A_{1}, B_{1}, C_{1}, D_{1}$ in the following way (see also Murty et al.[15]; Sattar [6]; Shamsul and Sattar [8]; Shamsul [13]; Shamsul [22] for details).

$$
\begin{gathered}
e^{-\lambda t}\left(\frac{\partial}{\partial t}+\mu-\lambda\right) \frac{\partial^{2} C_{1}}{\partial t^{2}}=-c \sum_{i, j=0}^{\infty} F_{1}(d) e^{-(i \lambda+j \mu) t} \\
e^{-\lambda t}\left(\frac{\partial}{\partial t}+\mu-\lambda\right)\left(\frac{\partial^{2} B_{1}}{\partial t^{2}}+6 \frac{\partial C_{1}}{\partial t}\right)= \\
-b \sum_{i, j=0}^{\infty} F_{1}(d) e^{-(i \lambda+j \mu) t} \\
e^{-\lambda t}\left(\frac{\partial}{\partial t}+\mu-\lambda\right)\left(\frac{\partial^{2} A_{1}}{\partial t^{2}}+3 \frac{\partial B_{1}}{\partial t}+6 C_{1}\right)+ \\
e^{-\mu t}\left(\frac{\partial}{\partial t}+\lambda-\mu\right)^{3} D_{1}=-\sum_{i, j=0}^{\infty} F_{0}(d) e^{-(i \lambda+j \mu) t} \\
\left(\frac{\partial}{\partial t}+\lambda\right)^{3}\left(\frac{\partial}{\partial t}+\mu\right) u_{1}=-\sum_{i, j=0}^{\infty} F_{2}(d) e^{-(i \lambda+j \mu) t} \\
\left(a+b t+c t^{2}\right)^{2}-\cdots
\end{gathered}
$$

Solving the equation (8), we get the value

$$
C_{1}=\sum_{i, j=0}^{\infty} \frac{c F_{1}(d) e^{-((i-1) \lambda+j \mu) t}}{(i \lambda+(j-1) \mu)((i-1) \lambda+j \mu)^{2}}
$$

Substituting the value of $C_{1}$ from (12) into equation (9), we obtain

$$
\begin{aligned}
e^{-\lambda t}( & \left.\frac{\partial}{\partial t}+\mu-\lambda\right) \frac{\partial^{2} B_{1}}{\partial t^{2}}=-6 \\
& \sum_{i, j=0}^{\infty} \frac{c F_{1}(d) e^{-(i \lambda+j \mu) t}}{((i-1) \lambda+j \mu)(i \lambda+(j-1) \mu)} \\
& -\sum_{i, j=0}^{\infty} b F_{1}(d) e^{-(i \lambda+j \mu) t}
\end{aligned}
$$

Now solving equation (13), we obtain

$$
\begin{aligned}
B_{1}= & 6 \sum_{i, j=0}^{\infty} \frac{c F_{1}(d) e^{-((i-1) \lambda+j \mu) t}}{((i-1) \lambda+j \mu)^{3}(i \lambda+(j-1) \mu)^{2}} \\
& +\sum_{i, j=0}^{\infty} \frac{b F_{1}(d) e^{-((i-1) \lambda+j \mu) t}}{((i-1) \lambda+j \mu)^{2}(i \lambda+(j-1) \mu)}
\end{aligned}
$$

Now using the value of $C_{1}$ from (12) and $B_{1}$ from (14) into equation (10), we obtain

$$
\begin{aligned}
& e^{-\lambda t}\left(\frac{\partial}{\partial t}+\mu-\lambda\right) \frac{\partial^{2} A_{1}}{\partial t^{2}}+e^{-\mu t}\left(\frac{\partial}{\partial t}+\lambda-\mu\right)^{3} D_{1}=- \\
& 12 \sum_{i, j=0}^{\infty} \frac{c F_{1}(a, d) e^{-(i \lambda+j \mu) t}}{((i-1) \lambda+j \mu)^{2}}-3 \sum_{i, j=0}^{\infty} \frac{b F_{1}(a, d) e^{-(i \lambda+j \mu) t}}{((i-1) \lambda+j \mu)} \\
& -\sum_{i, j=0}^{\infty} F_{0}(a, d) e^{-(i \lambda+j \mu) t}
\end{aligned}
$$

Now we have only one equation (15) for obtaining the unknown functions $A_{1}$ and $D_{1}$, for finding the value of $A_{1}$ and $D_{1}$ equating the coefficient of $e^{-\lambda t}$ and $e^{-\mu t}$ from the equation (15).

Thus, the determination of the first order improved solution of the equation (1) is completed. It should be noted that the solution for higher order systems can also carried out in the same manner as has been carried out in this study.

\section{Example}

As an example of the above procedure, consider a fourth order weakly nonlinear system governed by the ordinary differential equation

$$
x^{(i v)}+k_{1} \dddot{x}+k_{2} \ddot{x}+k_{3} \dot{x}+k_{4} x=-\varepsilon x^{3}
$$

Here $f(x, \dot{x}, \ddot{x}, \dddot{x})=x^{3}$

Therefore,

$$
\begin{aligned}
f^{(0)} & =\left(a+b t+c t^{2}\right)^{0} d^{3} e^{-3 \mu t}+3\left(a+b t+c t^{2}\right)^{1} \\
& d^{2} e^{-(\lambda+2 \mu) t}+3\left(a+b t+c t^{2}\right)^{2} d e^{-(2 \lambda+\mu) t} \\
& +\left(a+b t+c t^{2}\right)^{3} e^{-3 \lambda t}
\end{aligned}
$$

Thus for equation (16), the equations (8) to (11) respectively become

$$
\begin{gathered}
e^{-\lambda t}\left(\frac{\partial}{\partial t}+\mu-\lambda\right) \frac{\partial^{2} C_{1}}{\partial t^{2}}=-3 c d^{2} e^{-(\lambda+2 \mu) t} \\
e^{-\lambda t}\left(\frac{\partial}{\partial t}+\mu-\lambda\right)\left(\frac{\partial^{2} B_{1}}{\partial t^{2}}+6 \frac{\partial C_{1}}{\partial t}\right)= \\
-3 b d^{2} e^{-(\lambda+2 \mu) t} \\
e^{-\lambda t}\left(\frac{\partial}{\partial t}+\mu-\lambda\right)\left(\frac{\partial^{2} A_{1}}{\partial t^{2}}+3 \frac{\partial B_{1}}{\partial t}+6 C_{1}\right) \\
+e^{-\mu t}\left(\frac{\partial}{\partial t}+\lambda-\mu\right)^{3} D_{1}=- \\
\left\{3 a d^{2} e^{-(\lambda+2 \mu) t}+d^{3} e^{-3 \mu t}\right\}
\end{gathered}
$$




$$
\begin{aligned}
\left(\frac{\partial}{\partial t}\right. & +\lambda)^{3}\left(\frac{\partial}{\partial t}+\mu\right) u_{1}=-\left\{a^{3}+3 a^{2} b t\right. \\
& +3\left(a b^{2}+a^{2} c\right) t^{2}+\left(b^{3}+6 a b c\right) t^{3} \\
& \left.+3\left(b^{2} c+a c^{2}\right) t^{4}+3 b c^{2} t^{5}+c^{3} t^{6}\right\} e^{-3 \lambda t} \\
& -\left\{3 a^{2} d+6 a b d t+3\left(b^{2} d+2 a c d\right) t^{2}\right. \\
& \left.+6 b c d t^{3}+3 c^{2} d t^{4}\right\} e^{-(2 \lambda+\mu) t}
\end{aligned}
$$

The solution of the equation (17) is

$$
C_{1}=(3 / 4) Q^{2} R c d^{2} e^{-2 \mu t}
$$

Consider $P=1 / \lambda, Q=1 / \mu, R=1 /(\lambda+\mu)$ and $S=1 /(3 \lambda-\mu)$

Substituting the value of $C_{1}$ from the equation (21) into the equation (18), we obtain

$$
B_{1}=(9 / 4) Q^{3} R c d^{2} e^{-2 \mu t}+(9 / 4) Q^{2} R b d^{2} e^{-2 \mu t}
$$

To separate the equation (19) for determining unknown functions $A_{1}$ and $D_{1}$, we equate the coefficient of $e^{-\lambda t}$ and $e^{-\mu t}$, we obtain

$$
\begin{aligned}
& e^{-\lambda t}\left(\frac{\partial}{\partial t}+\mu-\lambda\right) \frac{\partial^{2} A_{1}}{\partial t^{2}}=0 \\
& e^{-\mu t}\left(\frac{\partial}{\partial t}+\lambda-\mu\right)^{3} D_{1}=-(27 / 2) Q^{2} c d^{2} \\
& \quad e^{-(\lambda+2 \mu) t}-(27 / 2) Q b d^{2} e^{-(\lambda+2 \mu) t} \\
& +(9 / 2) Q^{2} c d^{2} e^{-(\lambda+2 \mu) t}-3 a d^{2} \\
& e^{-(\lambda+2 \mu) t}-d^{3} e^{-3 \mu t}
\end{aligned}
$$

The particular solutions of (23) and (24) respectively become

$$
\begin{aligned}
A_{1}= & 0 \\
D_{1}= & (9 / 4) Q^{5} c d^{2} e^{-(\lambda+\mu) t}+(9 / 16) \\
& Q^{4} b d^{2} e^{-(\lambda+\mu) t}+(3 / 8) Q^{3} a d^{2} e^{-(\lambda+\mu) t} \\
& +(3 \mu-\lambda)^{-3} d^{3} e^{-2 \mu t}
\end{aligned}
$$

The solution of the equation (20) for $u_{1}$ is

$$
\begin{aligned}
u_{1}= & \left\{r_{1} a^{3}+\left(r_{1} t+r_{2}\right) a^{2} b+\left(r_{1} t^{2}+r_{3} t+r_{4}\right)\right. \\
& \left(a b^{2}+a^{2} c\right)+\left(r_{1} t^{3}+r_{5} t^{2}+r_{6} t+r_{7}\right) \\
& \left(b^{3}+6 a b c\right)+\left(r_{1} t^{4}+r_{8} t^{3}+r_{9} t^{2}+r_{10} t\right. \\
& \left.+r_{11}\right)\left(b^{2} c+a c^{2}\right)+\left(r_{1} t^{5}+r_{12} t^{4}+r_{13} t^{3}\right. \\
& \left.+r_{14} t^{2}+r_{15} t+r_{16}\right) b c^{2}+\left(r_{1} t^{6}+r_{17} t^{5}\right. \\
& \left.\left.+r_{18} t^{4}+r_{19} t^{3}+r_{20} t^{2}+r_{21} t+r_{22}\right) c^{3}\right\} e^{-3 \lambda t} \\
& +\left\{r_{23} a^{2} d+\left(r_{23} t+r_{24}\right) a b d+\left(r_{23} t^{2}+r_{25} t\right.\right. \\
& \left.+r_{26}\right)\left(b^{2} d+2 a c d\right)+\left(r_{23} t^{3}+r_{27} t^{2}+r_{28} t\right. \\
& \left.+r_{29}\right) b c d+\left(r_{23} t^{4}+r_{30} t^{3}+r_{31} t^{2}+r_{32} t\right. \\
& \left.\left.+r_{33}\right) c^{2} d\right\} e^{-(\mu+2 \lambda) t}
\end{aligned}
$$

where $r_{1}=(3 / 8) P^{3} S, r_{2}=r_{1} \times\{(3 / 2) P-S\}$,

$$
\begin{gathered}
r_{3}=r_{1} \times(3 P-2 S), r_{4}=r_{1} \times\left(2 P^{2}-3 P S+2 S^{2}\right), \\
r_{5}=r_{1} \times\{-3 S+(9 / 2) P\}, r_{6}=r_{1} \times\left(6 S^{2}-9 S P+9 P^{2}\right), \\
r_{7}=r_{1} \times\left\{\begin{array}{l}
-6 S^{3}+9 P S^{2}-9 P^{2} S \\
+(25 / 2) P^{3}
\end{array}\right\},
\end{gathered}
$$

$$
\begin{gathered}
r_{8}=r_{1} \times(-4 S+6 P), r_{9}=r_{1} \times\left(12 S^{2}-18 P S+18 P^{2}\right), \\
r_{10}=r_{1} \times\left(\begin{array}{l}
-24 S^{3}+36 P S^{2} \\
-36 P^{2} S+30 P^{3}
\end{array}\right),
\end{gathered}
$$

$$
\begin{gathered}
r_{11}=r_{1} \times\left\{\begin{array}{l}
24 S^{4}-36 P S^{3}+36 P^{2} S^{2} \\
-30 P^{3} S+(45 / 2) P^{4}
\end{array}\right\}, \\
r_{12}=r_{1} \times\{-5 S+(15 / 2) P\}, \\
r_{13}=r_{1} \times\left(20 S^{2}-20 P S+30 P^{2}\right), \\
r_{14}=r_{1} \times\left(\begin{array}{l}
-60 S^{3}+90 P S^{2}-90 P^{2} S \\
+75 P^{3}
\end{array}\right),
\end{gathered}
$$$$
r_{15}=r_{1} \times\left\{\begin{array}{l}
120 S^{4}-180 P S^{3}+180 P^{2} S^{2} \\
-150 P^{3} S+(225 / 2) P^{4}
\end{array}\right\} \text {, }
$$

$$
\begin{aligned}
r_{16} & =r_{1} \times\left\{-120 S^{5}+180 P S^{4}-180 P^{2} S^{3}\right. \\
& \left.+150 P^{3} S^{2}-150 P^{4} S+(315 / 4) P^{5}\right\},
\end{aligned}
$$

$$
r_{17}=r_{1} \times\{-6 S+(9 / 2) P\},
$$

$$
r_{18}=r_{1} \times\left(30 S^{2}-45 P S+45 P^{2}\right) \text {, }
$$$$
r_{19}=r_{1} \times\left\{\begin{array}{l}
-120 S^{3}+180 P S^{2}-180 P^{2} S \\
+150 P^{3}
\end{array}\right\},
$$

$$
\begin{aligned}
r_{20}= & r_{1} \times\left\{\begin{array}{l}
360 S^{4}-540 P S^{3}+240 P^{2} S^{2} \\
-240 P^{3} S+(375 / 2) P^{4}
\end{array}\right\}, \\
r_{21}= & r_{1} \times\left\{-720 S^{5}+1080 P S^{4}-1080 P^{2} S^{3}\right. \\
& \left.+900 P^{3} S^{2}-670 P^{4} S+(945 / 2) P^{5}\right\},
\end{aligned}
$$$$
r_{22}=r_{1} \times\left\{720 S^{6}-1080 P S^{5}+1080 P^{2} S^{4}\right.
$$$$
-900 P^{3} S^{3}+675 P^{4} S^{2}-(945 / 2)
$$$$
\left.P^{5} S+315 P^{6}\right\} \text {, }
$$

$r_{23}=-(3 / 2) P R^{3}, r_{24}=r_{23} \times(3 R+P)$, 


$$
\begin{gathered}
r_{25}=r_{23} \times(6 R+P), r_{26}=r_{23} \times\left(12 R^{2}+P R+2 P^{2}\right), \\
r_{27}=r_{23}\{(3 / 2) P+9 R\}, \\
r_{28}=r_{23} \times\left\{(3 / 2) P^{2}+9 P R+9 R^{2}\right\}, \\
r_{29}=r_{23} \times\left\{\begin{array}{l}
(3 / 4) P^{3}+(9 / 2) P^{2} R+ \\
9 P R^{2}+60 R^{3}
\end{array}\right\}, \\
r_{30}=r_{23} \times\{2 P+12 R\}, r_{31}=r_{23} \times\left(3 R^{2}+18 P R+72 P^{2}\right), \\
r_{32}=r_{23} \times\left\{\begin{array}{l}
3 P^{3}+18 P^{2} R+72 P R^{2} \\
+240 R^{3}
\end{array}\right\}, \\
r_{33}=r_{23} \times\left\{\begin{array}{l}
(3 / 2) P^{4}+9 P^{3} R+36 P^{2} R^{2} \\
+120 P R^{3}+360 R^{4}
\end{array}\right\}
\end{gathered}
$$

Substituting the values of $A_{1}, B_{1}, C_{1}$ and $D_{1}$ from the equations (25), (22), (21) and (26) into equation (19) and integrating, we obtain

$$
\begin{aligned}
a= & a_{0} \\
b= & b_{0}+\varepsilon\left(9 Q^{3} R / 8\right)\left(Q c_{0} d_{0}^{2}+b_{0} d_{0}^{2}\right) \\
& \left(1-e^{-2 \mu t}\right) \\
c= & c_{0}+\varepsilon\left(3 Q^{3} R / 8\right) c_{0} d_{0}^{2}\left(1-e^{-2 \mu t}\right) \\
d= & d_{0}+\varepsilon\left[Q ^ { 3 } R \left\{(9 / 4) Q^{2} c_{0} d_{0}^{2}+(9 / 16)\right.\right. \\
& Q b_{0} d_{0}^{2}+(3 / 8) a_{0} d_{0}^{2}\left(1-e^{-(\lambda+\mu) t}\right) \\
& \left.+(2 \mu)^{-1}(3 \mu-\lambda)^{-3} d_{0}^{3}\left(1-e^{-2 \mu t}\right)\right]
\end{aligned}
$$

Therefore, we obtain the first approximate solution of the equation (16) as

$$
\begin{aligned}
x(t, \varepsilon)= & \left(a+b t+c t^{2}\right) e^{-\lambda t}+d e^{-\mu t} \\
& +\varepsilon u_{1}(a, b, c, d, t)+\cdots
\end{aligned}
$$

where $a, b, c$ and $d$ are given by the equations (28) to (31) and $u_{1}$ is given by (27).

\section{Results and Discussion}

To make sure the efficiency of our results, we have compared our results to the numerical results obtained by fourth order Runge-Kutta method for the different set of initial conditions.

First of all, $x(t, \mathcal{E})$ has been computed from (32) by considering values of $\lambda=3.0, \mu=0.21$ in which $a, b, c$ and $d$ are calculated from equations (28) to (31) with the initial conditions $a_{0}=0.30, b_{0}=0.10, \quad c_{0}=0.05$ and $d_{0}=0.25$ when $\varepsilon=0.1$, Fig. 1 represents the perturbation results which are plotted by the continuous line and the corresponding numerical solution has been computed by a fourth-order Runge-Kutta method, which are plotted by a dotted line as follows:

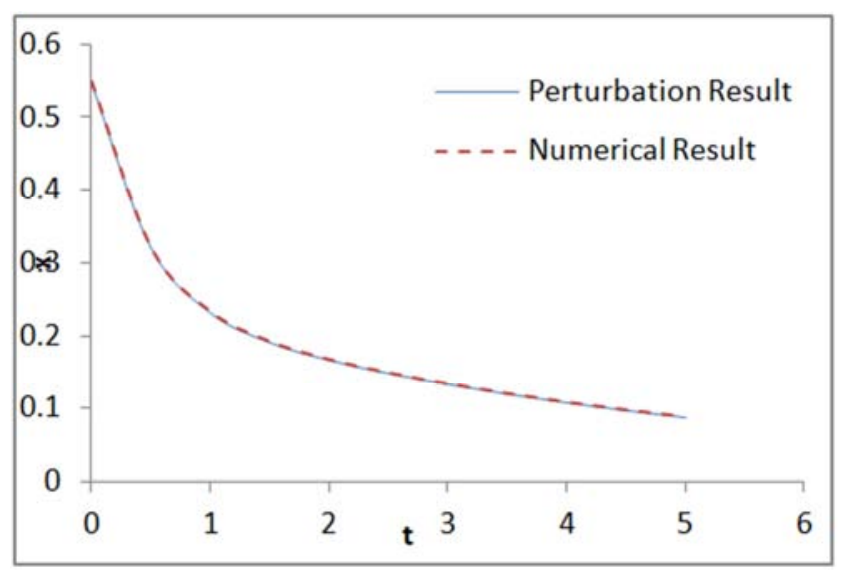

Fig. 1. Perturbation results are plotted by continuous line and numerical results are plotted by dotted line.

Secondly, $x(t, \varepsilon)$ has been computed from (32) by considering values of $\lambda=4.0, \mu=0.65$ in which $a, b, c$ and $d$ are calculated from equations (28) to (31) with the initial conditions $a_{0}=0.20, b_{0}=0.30, \quad c_{0}=0.20$ and $d_{0}=0.40$ when $\varepsilon=0.1$. Fig. 2 represents the perturbation results which are plotted by the continuous line and the corresponding numerical solution has been computed by a fourth-order Runge-Kutta method, which are plotted by a dotted line as follows:

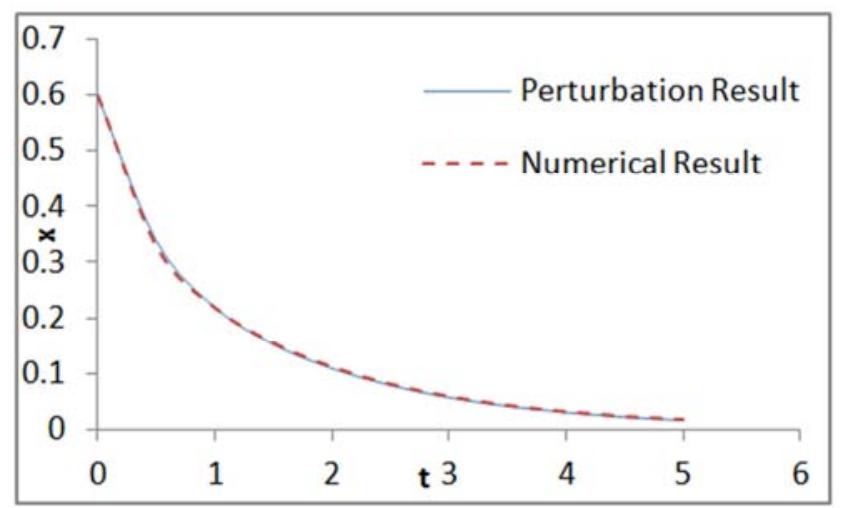

Fig. 2. Perturbation results are plotted by continuous line and numerical results are plotted by dotted line.

Finally, $x(t, \varepsilon)$ has been computed from (32) by considering values of $\lambda=4.0, \mu=0.30$ in which $a, b, c$ and $d$ are calculated from equations (28) to (31) with the initial conditions $a_{0}=0.30, b_{0}=0.20, c_{0}=0.10$ and $d_{0}=0.40$ when $\varepsilon=0.1$. Fig. 3 represents the perturbation results which are plotted by the continuous line and the 
corresponding numerical solution has been computed by a fourth-order Runge-Kutta method, which are plotted by a dotted line as follows:

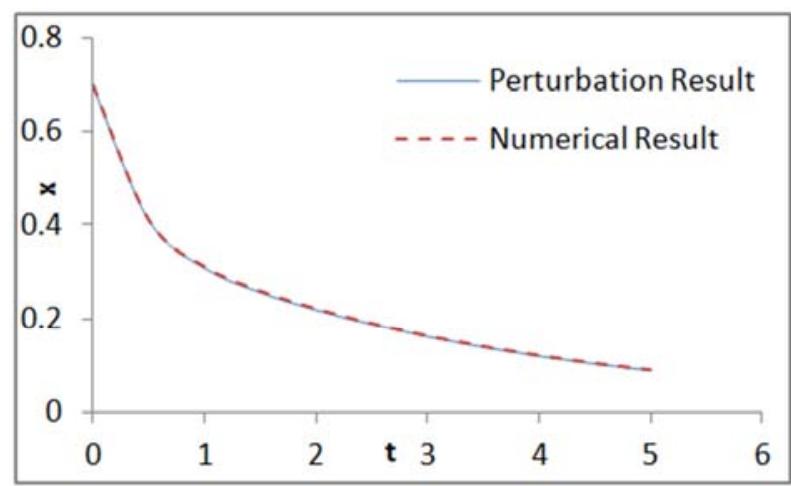

Fig. 3. Perturbation results are plotted by continuous line and numerical results are plotted by dotted line.

\section{Conclusion}

Based upon the KBM method of fourth order more critically damped nonlinear systems, we have been able to obtain an analytical approximate solution in this study. Moreover, we have shown in this study that the results obtained by the proposed method correspond satisfactorily to the numerical results obtained by the fourth order RungeKutta method. It is, therefore, concluded that the modified KBM method provides highly accurate results, which can be applied for different kinds of nonlinear differential systems.

\section{Acknowledgement}

The authors are grateful to Mr. Md. Mizanur Rahman, Associate Professor, Department of Mathematics, Islamic University, Bangladesh, for his invaluable comments on the early draft of this paper. The authors are also thankful to $\mathrm{Mr}$. Md. Imamunur Rahman for his assistance in editing this paper.

\section{References}

[1] Bogoliubov, N. N. and Mitropolskii, Y. A., Asymptotic Methods in the Theory of Nonlinear Oscillations, Gordan and Breach, New York, 1961.

[2] Krylov, N. N. and Bogoliubov, N. N., Introduction to Nonlinear Mechanics, Princeton University Press, New Jersey, 1947.

[3] Popov, I. P., "A Generalization of the Bogoliubov Asymptotic Method in the Theory of Nonlinear Oscillations (in Russian)", Dokl. Akad. USSR, vol. 3, pp. 308-310, 1956.

[4] Mendelson, K. S., "Perturbation Theory for Damped Nonlinear Oscillations", J. Math. Physics, vol. 2, pp. 3413$3415,1970$.

[5] Murty, I. S. N., and Deekshatulu, B. L., "Method of Variation of Parameters for Over-Damped Nonlinear Systems", J. Control, vol. 9(3), pp. 259-266, 1969.
[6] Sattar, M. A., “An asymptotic Method for Second Order Critically Damped Nonlinear Equations", J. Frank. Inst., vol. 321, pp. 109-113, 1986.

[7] Murty, I. S. N., "A Unified Krylov-Bogoliubov Method for Solving Second Order Nonlinear Systems", Int. J. Nonlinear Mech. vol. 6, pp. 45-53, 1971.

[8] Osiniskii, Z., "Longitudinal, Torsional and Bending Vibrations of a Uniform Bar with Nonlinear Internal Friction and Relaxation," Nonlinear Vibration Problems, vol. 4, pp. 159166, 1962.

[9] Mulholland, R. J., "Nonlinear Oscillations of Third Order Differential Equation," Int. J. Nonlinear Mechanics, vol. 6. Pp. 279-294, 1971.

[10] Bojadziev, G. N., "Damped Nonlinear Oscillations Modeled by a 3-dimensional Differential System", Acta Mechanica, vol. 48 , pp. 193-201, 1983.

[11] Sattar, M. A., "An Asymptotic Method for Three-dimensional Over-damped Nonlinear Systems", Ganit, J. Bangladesh Math. Soc., vol. 13, pp. 1-8, 1993.

[12] Shamsul, M. A., "Asymptotic Methods for Second Order Over-damped and Critically Damped Nonlinear Systems", Soochow Journal of Math., vol. 27, pp. 187-200, 2001.

[13] Shamsul, M. A., "On Some Special Conditions of Third Order Over-damped Nonlinear Systems", Indian J. Pure Appl. Math., vol. 33, pp. 727-742, 2002.

[14] Akbar, M. A., Paul, A. C. and Sattar, M. A., "An Asymptotic Method of Krylov-Bogoliubov for Fourth Order Over-damped Nonlinear Systems", Ganit, J. Bangladesh Math. Soc., vol. 22, pp. 83-96, 2002.

[15] Murty, I. S. N., "Deekshatulu, B. L. and Krishna, G., "On an Asymptotic Method of Krylov-Bogoliubov for Over-damped Nonlinear Systems", J. Frank. Inst., vol. 288, pp. 49-65, 1969.

[16] Akbar, M. A., Shamsul, M. A. and Sattar M. A. "Asymptotic Method for Fourth Order Damped Nonlinear Systems", Ganit, J. Bangladesh Math. Soc., Vol. 23, pp. 41-49, 2003.

[17] Akbar, M. A., Shamsul, M. A. and Sattar M. A., "KrylovBogoliubov-Mitropolskii Unified Method for Solving $n$-th Order Nonlinear Differential Equations Under Some Special Conditions Including the Case of Internal Resonance", Int. J. Non-linear Mech, Vol. 41, pp. 26-42, 2006.

[18] Akbar, M. A., Uddin, M. S., Islam, M. R. and Soma, A. A. "Krylov-Bogoliubov-Mitropolskii (KBM) Method for Fourth Order More Critically Damped Nonlinear Systems", J. Mech. of Continua and Math. Sciences, Vol. 2(1), pp. 91-107, 2007.

[19] Islam, M. R., Rahman, M. H. and Akbar, M. A. "An Analytical Approximate Solution of Fourth Order More Critically Damped Nonlinear Systems", Indian Journal of Mathematics, Vol. 50(3), pp. 611-626, 2009.

[20] Hakim, M. A. "On Fourth Order More Critically Damped Nonlinear Differential Systems", Journal of Physical Science, Vol. 15, pp.113-127, 2011.

[21] Akbar, M. A., Shamsul, M. A. and Sattar, M. A. "A Simple Technique for Obtaining Certain Over-damped Solutions of an n-th Order Nonlinear Differential Equation”, Soochow Journal of Mathematics, Vol. 31(2), pp. 291-299, 2005. 